\title{
Brain Activity Supporting Working Memory Accuracy in Patients with Paranoid Schizophrenia: A Functional Magnetic Resonance Imaging Study
}

\author{
Claudia Wolf ${ }^{a}$ Stefanie Linden ${ }^{a, b}$ Margaret C. Jackson ${ }^{a}$ David Healy ${ }^{b}$ \\ Alison Baird ${ }^{c}$ David E.J. Linden ${ }^{a, b}$ Johannes Thome ${ }^{c, d}$ \\ aWolfson Centre for Cognitive and Clinical Neuroscience, School of Psychology, Bangor University, Bangor, and

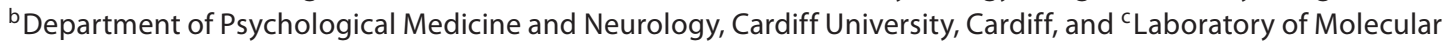 \\ Psychiatry and Pharmacology, Institute of Life Science, School of Medicine, Swansea University, Swansea, UK; \\ ${ }^{\mathrm{d} D e p a r t m e n t}$ of Psychiatry and Psychotherapy, Rostock University, Rostock, Germany
}

\section{Key Words}

Functional magnetic resonance imaging $\cdot$ Lateral prefrontal cortex - Occipitotemporal cortex $\cdot$ Performance matching • Hyperactivation $\cdot$ Faces $\cdot$ Working memory $\cdot$ Schizophrenia nition in high-functioning patients with hypofrontality is explained by activation of contralateral homologue areas combined with enhanced recruitment of sensory areas.

Copyright $\odot 2011$ S. Karger AG, Basel

\section{Introduction}

Among the cognitive functions frequently affected in schizophrenia, working memory (WM) has been recognized as one of the most consistent deficits [1,2]. Reduced WM accuracy for faces and emotional face expressions has been observed in patients with schizophrenia compared to healthy participants $[3,4]$.

There is an ongoing debate [5-7] about whether pathological changes are reflected in alterations of the BOLD response in frontoparietal WM networks [8]. Against this, it has been argued that activity differences between groups are confounded by differences in task performance and other factors such as level of education. Recent studies that addressed this issue by matching groups on task performance reported WM performance-dependent [9] and -independent [10] activity differences between patients and controls in WM-related regions. We

\section{KARGER}

Fax +4161306 1234 E-Mail karger@karger.ch www.karger.com

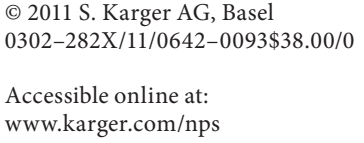

Prof. David Linden

MRC Centre for Neuropsychiatric Genetics and Genomics

Sir Henry Wellcome Building, Cardiff University School of Medicine Cardiff CF14 4XN (UK)

Tel. +442920687 928, E-Mail linden@ cardiff.ac.uk 
investigated whether emotional face WM-related neural network activity differs between high-performing patients with mainly paranoid schizophrenia and healthy participants. In particular we wanted to probe whether activity differences between groups in disease-associated areas (e.g. prefrontal cortex, PFC) would persist under these conditions. For this reason we included only clinically stable and homogeneous patients with at most mild cognitive impairments, good task performance, and matched patients with controls for additional confounding factors. Our results revealed a frontooccipitotemporal network that supported WM performance in patients.

\section{Methods}

\section{Participants}

Ten outpatients and 2 inpatients diagnosed with schizophrenia spectrum disorder (1 schizoaffective, 11 paranoid schizophrenia) according to DSM-IV criteria were assessed with the Structured Clinical Interview for DSM-IV and recruited by a psychiatrist (S.L.) from the Psychiatry Unit at Gwynedd Hospital. Current clinical symptoms were evaluated with the Positive and Negative Symptom Scales [11] and the premorbid IQ using the National Adult Reading Test. An equal number of healthy volunteers matched for gender, handedness, ethnicity, age and education were selected from a large control data sample for the same functional magnetic resonance imaging (fMRI) paradigm [12]. Control participants had no lifetime or family history of psychiatric or neurological disease. Patients and controls had normal or corrected to normal vision. They provided written informed consent prior to participation and were paid GBP 25. The study was approved by the ethics committees at the School of Psychology, Bangor University and at the North Wales NHS-Trust.

\section{Stimuli}

Six adult, male, greyscale face images each displaying neutral, happy and angry expressions were used [13]. Each image covered approximately $1.43^{\circ} \times 1.36^{\circ}$. Scrambled greyscale face images selected at random were displayed to cover the face locations during encoding of fewer than 4 faces.

\section{WM Task for Emotional Faces}

In an event-related design we investigated visual WM for emotional faces and task-related brain activity through the manipulation of face expression (angry, happy, and neutral) and the number of faces to be remembered (load 1, 2, 3, 4). Each of the 12 conditions consisted of 4 match and 4 non-match trials. Trials were distributed over 4 runs with 48 trials each to minimize fatigue effects. Face expressions and number of faces varied randomly between trials and type of face expression was kept constant within one trial. All trials started with fixation towards a central cross on the display, which served as baseline. This was followed by a 2 -second presentation of the memory array, a delay of $1 \mathrm{~s}$, and the test face, where participants had to indicate a match or nonmatch re- sponse via the respective button. The between-trials fixation interval jittered between 4,500 and 6,000 ms. A detailed description of design can be found in Jackson et al. [14].

Acquisition and Analysis of Behavioural and Imaging Data

The task was generated and behavioural data recorded with the E-Prime software [version $1.1 ; 15]$. Scanning was performed with a Philips 1.5 T MRI whole-body scanner with a SENSE parallel head coil. Blood oxygenation level-dependent images were acquired by using a $\mathrm{T}_{2}{ }^{*}$-weighted gradient echo-planar sequence. During each of the four WM sessions 343 volumes were acquired. For the co-registration with functional images one high-resolution $\mathrm{T}_{1}$-weighted three-dimensional volume was acquired. For further details regarding the imaging parameters please refer to Jackson et al. [14].

WM accuracy was assessed by calculating $\mathrm{d}^{\prime}$ values $\left(\mathrm{d}^{\prime}=\mathrm{z}-\right.$ transformed hits - z-transformed false alarms) for each of the 12 conditions. WM capacity for faces was measured by individual Cowan's $\mathrm{K}$ max values for each emotion [Cowan's $\mathrm{K}$ max $=$ maximal $\mathrm{K}$ reached for this individual at any array size; Cowan's $\mathrm{K}$ values $=$ array size $\times($ hits - FA)] [16].

Imaging data analysis was performed using the BrainVoyager 1.9.10 software (Braininnovation, Maastricht, The Netherlands). Functional images were co-registered with the structural threedimensional image, spatially normalized to the Talairach system [17] and resampled at a voxel size of $1 \times 1 \times 1 \mathrm{~mm}^{3}$, resulting in $56 \mathrm{z}$-normalized volume time course files (eight runs could not be used because of motion artefacts or chance performance head motion $>3 \mathrm{~mm}$ or chance performance; FA mean $>0.5$ ). Functional images were scan time-corrected using sinc interpolation, three-dimensional motion-corrected using trilinear interpolation, spatially smoothed (8-mm Gaussian kernel), and temporally high pass-filtered (3 cycles per time course). The general linear model (GLM) of the experiment was computed with predictors for each of the 12 conditions for all correct trials, one separate predictor for all error trials and 6 predictors derived from the head motion correction for each subject. All but the motion predictors were convolved with a two-gamma haemodynamic reference function.

We computed a random effect (RFX-GLM), to obtain beta values per subject and condition at each voxel. These were used as dependent variable to compute a second-level RFX-mixed 3-factors ANOVA with the within-subject factors emotion (3 levels), load (4 levels) and the between-subject factor group (2 levels) to generate functional whole-brain three-dimensional maps for the main effect of group, the group $\times$ emotion interaction and the contrast load 4 minus 1 . Clusters of activation were determined using a voxel-wise threshold at $\mathrm{p}<0.01$ significance level and a cluster-based threshold of 200 voxels for the main effect of group in order to minimize false positive and false negative effects. The interaction between group $\times$ emotion was thresholded at $\mathrm{p}<0.05$ and 1,500 voxels. The contrast load 4 minus 1 was thresholded at $\mathrm{p}<0.05$ and 500 voxels. The cluster thresholds were calculated with Brainvoyager QX Cluster-level Statistical Threshold estimator based on a Monte Carlo simulation with 1,000 iterations. For each of the obtained clusters an RFX-GLM region of interest (ROI) analysis was computed to extract beta values representing the mean activity over the entire cluster for all 12 task conditions (including only correct trials) per subject for extended statistical analysis. 


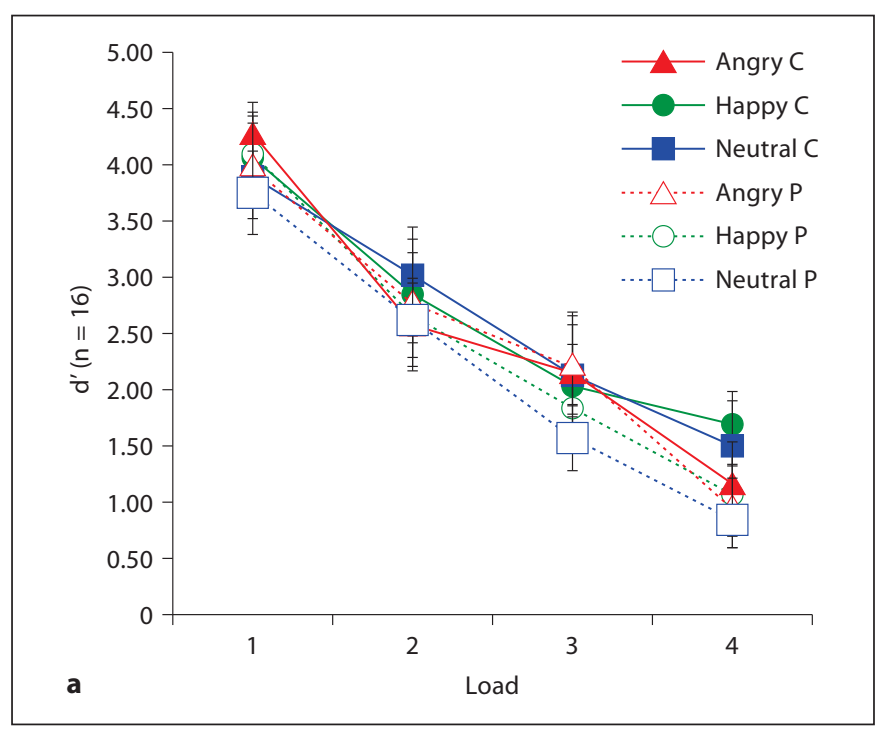

Fig. 1. a Comparison of $\mathrm{d}^{\prime}$ (WM accuracy) means for each emotion (angry, happy, and neutral) at each load (1-4) between controls $(\mathrm{C})$ and patients with schizophrenia $(\mathrm{P})$ showed no significant differences $(p>0.05)$. WM accuracy significantly $(p<0.001)$ decreased with increasing load. Error bars show the \pm SEM.

\section{Statistical Analysis}

Matching of Patients and Controls. Independent-samples $t$ tests were used to assess whether controls and patients differed according to age and education.

Group Effects on WM Performance. Mixed ANOVA [betweensubject factor: group (controls, patients); within-subject factors: emotion (angry, happy, neutral) and load (1-4)] was used to test the effect of emotion, load, group and possible interactions on accuracy of WM for emotional faces. Independent-samples $t$ test was calculated to test for a group effect on load 4 (averaged across emotions). We performed another mixed ANOVA [between-subject factor: group (controls, patients); within-subject factor maximal Cowan's K values (all 3 emotions)] to assess group effects on the individual WM capacity for each emotion.

Group Effects on Brain Activity. Mixed ANOVAs with two within-subject factors (emotion: angry, happy, neutral and load: 1-4) and one between-subject factor (group: controls, patients) were calculated to specify the strength of effects on brain activation for each cluster. We then tested the group and load effect on beta means for each load (averaged across emotion) using mixed ANOVAs with the within-subject factor (load: 1-4) and one betweensubject factor (group: controls, patients). Group effects on beta means for each load averaged across emotions were analysed with two-tailed independent-sample $t$ tests to identify at which loads groups differed. For the interaction between group and emotion, group effects on beta mean values for each emotion averaged across loads were analysed with two-tailed independent-sample $t$ tests to analyse how groups differed for each emotion. We also used twotailed independent-sample $t$ tests to compare between groups the percentage BOLD-signal change averaged across all 12 conditions for each time point. All $t$ test results were Bonferroni-corrected.

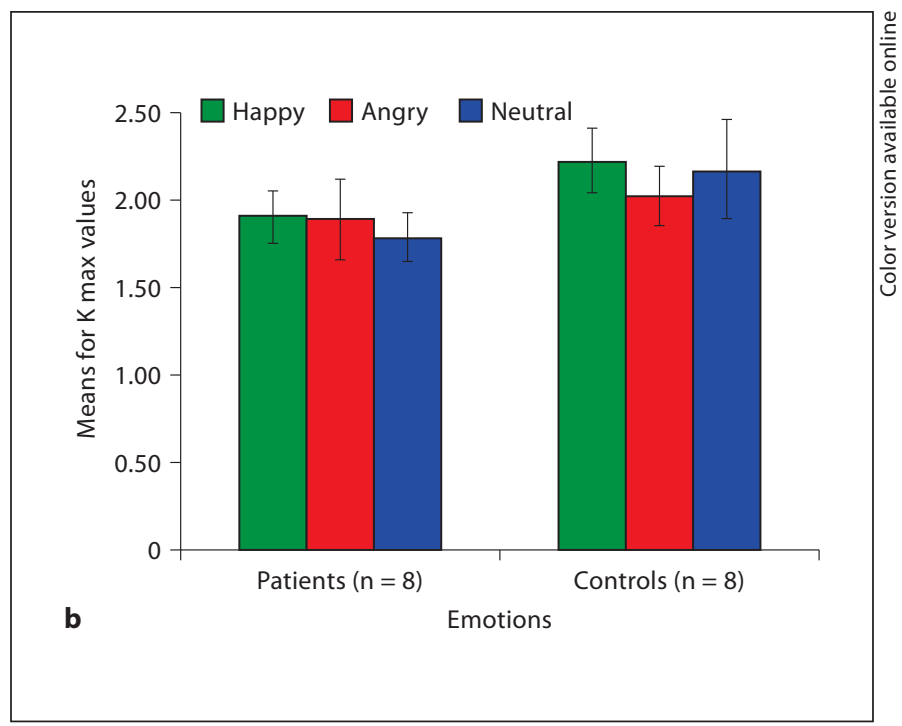

b Comparison of K max mean values (WM capacity) for happy, angry and neutral faces between patients with schizophrenia and controls showed no significant differences. There were no significant differences between WM-capacities for different emotional faces. Error bars show the \pm SEM.

\section{Results}

\section{Behavioural Data}

Data from 4 patients (2 inpatients) had to be excluded due to head movement artefacts and/or chance task performance. The clinical parameters for the remaining patients and matching details for patients and controls are shown in online supplementary tables 1 and 2 (www. karger.com/doi/10.1159/000323800).

Our patients who were all except 1 diagnosed with paranoid schizophrenia showed no significant performance deficits of WM for emotional faces compared to healthy volunteers.

The mixed ANOVA [between-subject factor: group (controls, patients); within-subject factors: emotion (angry, happy, neutral) and load (1-4)] for mean accuracy $\left(d^{\prime}\right)$ of WM for emotional faces comparing controls and patients revealed a main effect of load $\mathrm{F}(3,42)=84.19$, $\mathrm{p}<0.001$ (fig. 1a), but no effects of group or emotion and no two- or three-way interaction ( $\mathrm{p}>0.05)$.

The mixed ANOVA [between-subject factor: group (controls, patients); within-subject factor: emotion (angry, happy, neutral)] for K max values (WM capacity) comparing controls and patients revealed only non-significant $(p>0.05)$ results for main effects and the interaction (fig. 1b). 


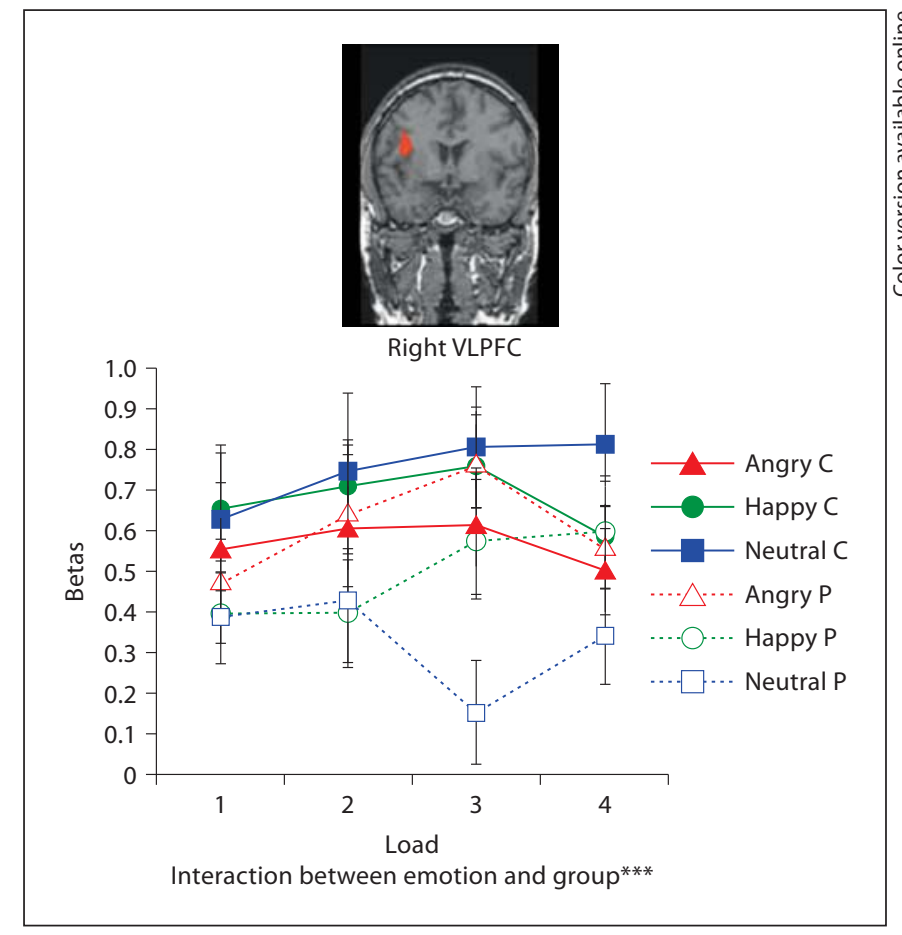

Fig. 2. The location of the ROI cluster for the emotion and group interaction ( $\mathrm{p}<0.05$ and cluster threshold 1,500 voxels) and beta values for each emotion at each load are shown. Beta mean values for each emotion averaged across loads revealed that this interaction was driven by significantly ( $\mathrm{p}<0.05$, Bonferroni-corrected) lower activity for neutral faces in patients compared to controls. Error bars show the \pm SEM.

\section{Imaging Data}

Only correct trials were included in the analysis of BOLD response to compare WM accuracy-related areas between patients and controls. There was a significant interaction between emotion and group in the right ventrolateral PFC (VLPFC, fig. 2, online suppl. table 5). This effect was driven by lower activity for neutral faces in patients compared with control participants.

We found a main effect for group (fig. 3, 4, online suppl. table 3 ) in the left occipitotemporal cortex (OTC) and lateral PFC (LPFC, driven by higher activation for patients), and right LPFC and medial PFC (MPFC, driven by higher activation in controls). Post-hoc tests revealed that this main effect of group on beta means for load was driven by significantly increased activity in patients compared to controls at load 2 in the left OTC ( $p<0.01$, Bonferroni-corrected), and LPFC ( $\mathrm{p}<0.05$, Bonferroni-corrected). The main effect of group was driven by significantly lower activity in patients compared to controls at load 3 ( $p<0.05$, Bonferroni-corrected) in the MPFC ( $p<$
0.05, Bonferroni-corrected) and right LPFC ( $\mathrm{p}<0.01$, Bonferroni-corrected). The MPFC also showed a significant effect of load, as did right and left parietal cortex (fig. 4, online suppl. table 4).

Event-related averaging showed the maximal BOLDsignal peak $8 \mathrm{~s}$ after the onset of encoding in the right LPFC in controls and in the left LPFC in patients while there was neither a clear peak response in the right LPFC in patients nor in the left LPFC in controls (fig. 3). In the left OTC patients showed the maximum BOLD signal $6 \mathrm{~s}$ after encoding onset while controls showed an earlier and smaller peak after $4 \mathrm{~s}$ (fig. 3). In all load-sensitive areas both groups showed BOLD-signal peaks $8 \mathrm{~s}$ after the onset of encoding except for the right parietal cortex in controls where BOLD signal peaked $6 \mathrm{~s}$ after onset of encoding (fig. 4).

Activity in the OTC (fig. 5, online suppl. table 6) differed less between patients and controls in the early phase of encoding (reflected in the time point 13, thus $4 \mathrm{~s}$ after onset of sample presentation), than during the later stages of the task (most significant differences at time point 17). In the left LPFC (fig. 5, online suppl. table 6) activity differed between patients and controls solely during the later stages of the task (most significant differences at time point 17).

\section{Discussion}

\section{Network Activity Supporting WM in Patients}

Patients with mainly paranoid schizophrenia compared to control participants showed decreased activity in the right LPFC and load-sensitive MPFC. This was contrasted by left lateralized increased activity in the lateralprefrontal and the occipital-temporal region in patients compared to controls. Activation of the right lateral PFC and in particular right VLPFC for face WM has been described for healthy populations [14, 18-20]. Activation of the left PFC has been shown to support task performance with increasing WM load in healthy volunteers [21]. Dysfunction of the right LPFC in patients, which also has been reported recently by another study [22], might thus result in compensatory recruitment of the left LPFC to support face WM. However, without longitudinal studies and/or normative data, such interpretation in terms of compensatory network activation has to remain preliminary. Compensatory activation of the left LPFC in schizophrenia has been suggested by several studies [23-25]. A longitudinal study found improved WM accuracy in patients after several weeks of clinical intervention, which was associated 


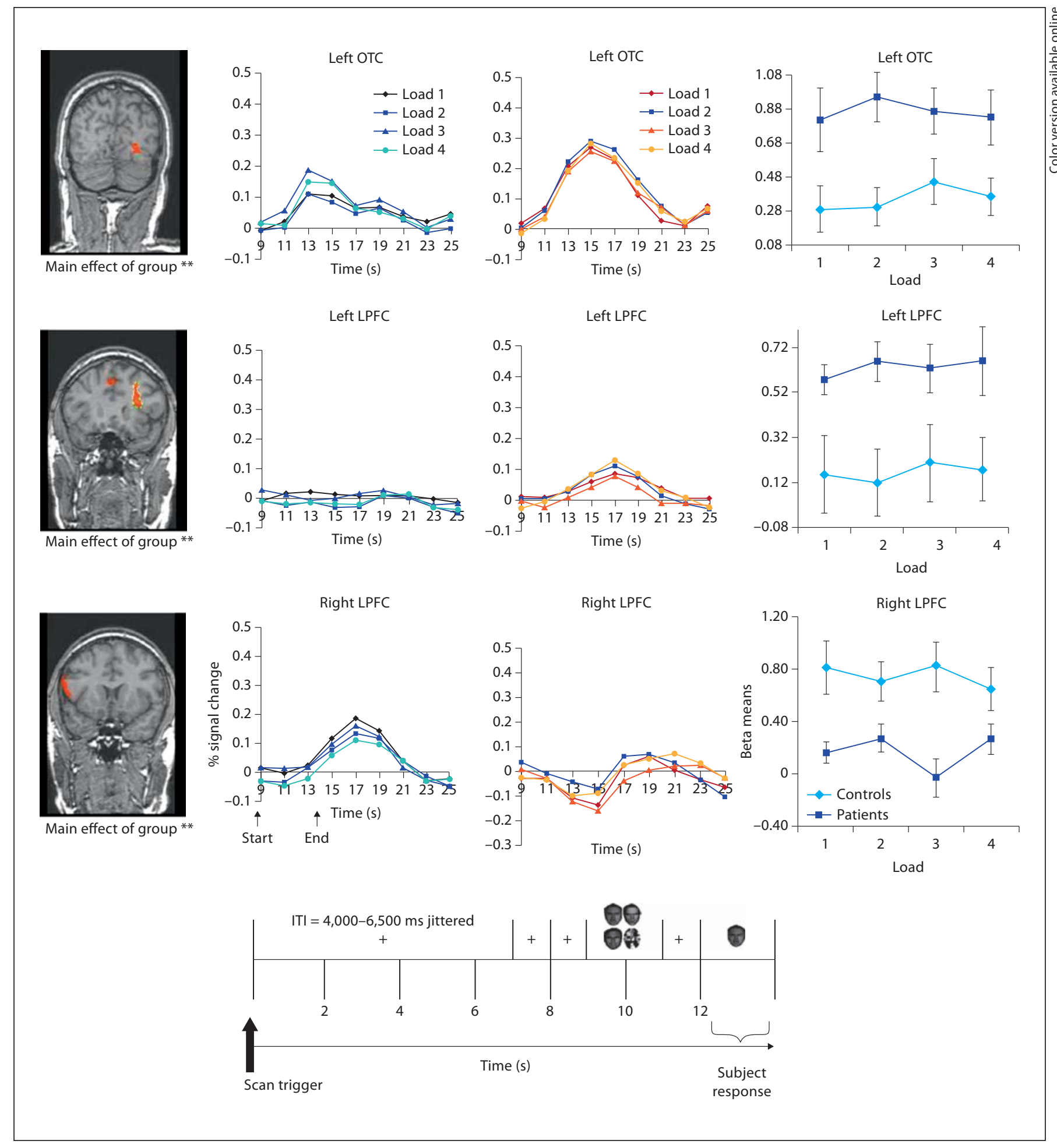

Fig. 3. The location of the ROI clusters for the main effect of group ( $p<0.01$ and cluster threshold 200 voxels), percentage BOLDsignal change mean values (arrows indicate timing of stimulus presentation from onset of the sample display until offset of the test face $5 \mathrm{~s}$ later - the time course thus captures the duration of one full trial) and beta mean values averaged for each load across emotions are shown. Beta means for load were significantly $(\mathrm{p}<$ 0.01 ) higher in patients with schizophrenia compared to control participants in the left occipital-temporal cortex and left lateral PFC. Beta means for load were significantly $(\mathrm{p}<0.01)$ lower in patients compared to controls in the right lateral PFC. Error bars show the \pm SEM. 


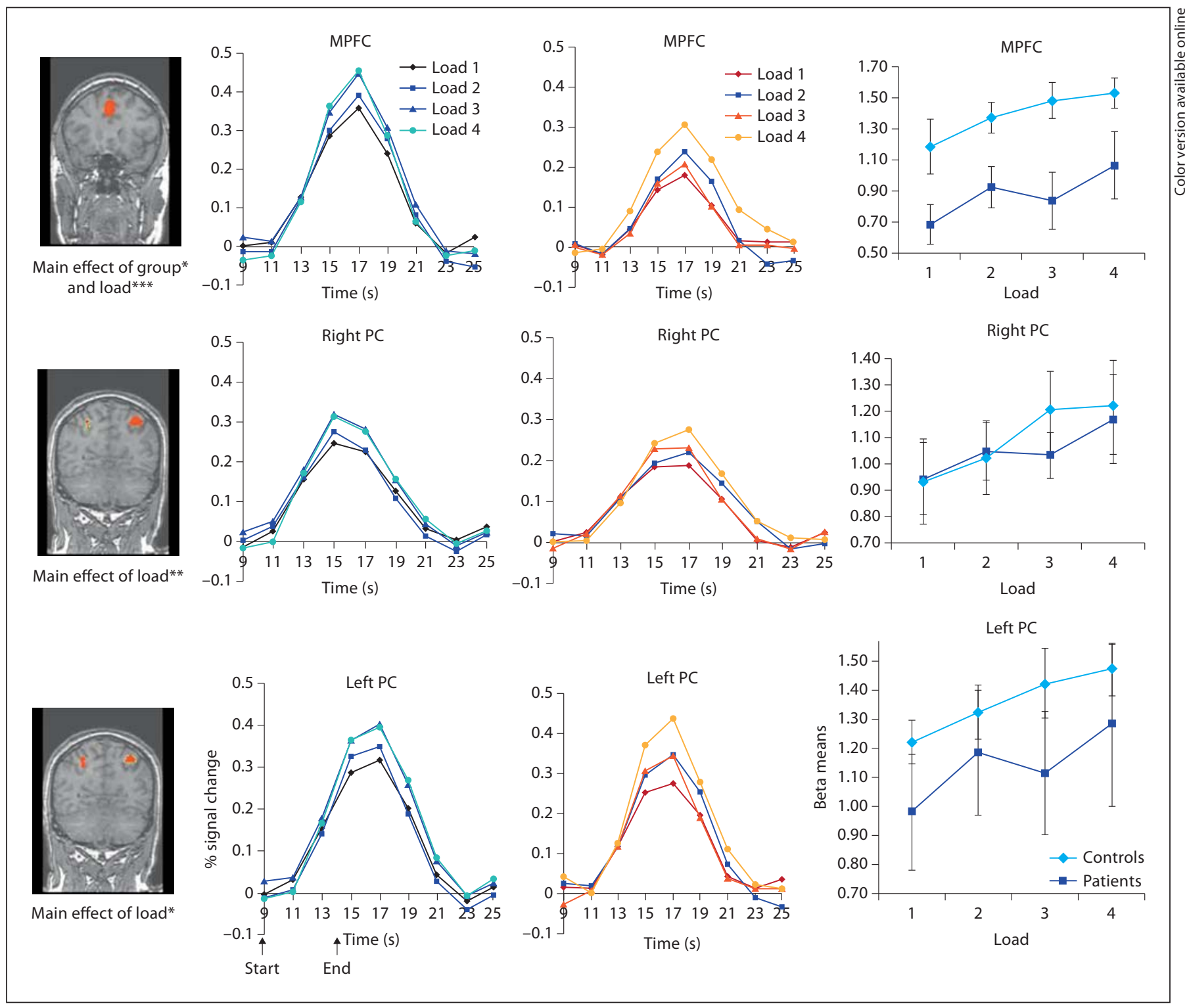

Fig. 4. The location of the ROI clusters for the contrast load $4 \mathrm{mi}-$ nus 1 ( $\mathrm{p}<0.05$ and cluster threshold 500 voxels), percentage BOLD-signal change mean values and beta mean values averaged for each load across emotions are shown. Beta means for load increased with increasing load in the right $(\mathrm{p}<0.01)$ and left $(\mathrm{p}<$
$0.05)$ parietal cortex and in the MPFC $(\mathrm{p}<0.001)$. Additionally in the MPFC, beta means for load were significantly lower $(\mathrm{p}<0.05)$ in patients with schizophrenia compared to control participants. Error bars show the \pm SEM. with enhanced activation within frontal-temporal regions [26]. Karlsgodt et al. [25] suggested that the degree of hyperfrontality could indicate the ability for compensatory adaptations in the high-performing patients.

However, our findings of decreased right and increased left PFC activation in patients compared to controls could also be specific to the type of stimuli used in the study. Lower activation of the left PFC or loss of the left domi- nance effect has been observed for verbal WM in patients with schizophrenia compared to controls [27, 28]. Other studies have found no activity differences in the left PFC between patients and controls during verbal [29-31] and face WM [31]. Interestingly one of these studies found also decreased activation of the right PFC at higher levels of task difficulty in patients versus controls [9]. However, patients performed significantly worse than controls in all 
Fig. 5. Comparison of percentage signal change means (across all conditions) between groups for each time point in the left OTC and LPFC revealed significant ( $<<$ 0.001 , Bonferroni-corrected) group differences with the maximal difference at $17 \mathrm{~s}$. Note that the difference in the left OTC started to become significant at $13 \mathrm{~s}$ (thus, $4 \mathrm{~s}$ after onset of the sample array, reflecting the haemodynamic delay of first-pass neural processing) and in the left LPFC $2 \mathrm{~s}$ later at $15 \mathrm{~s}$.

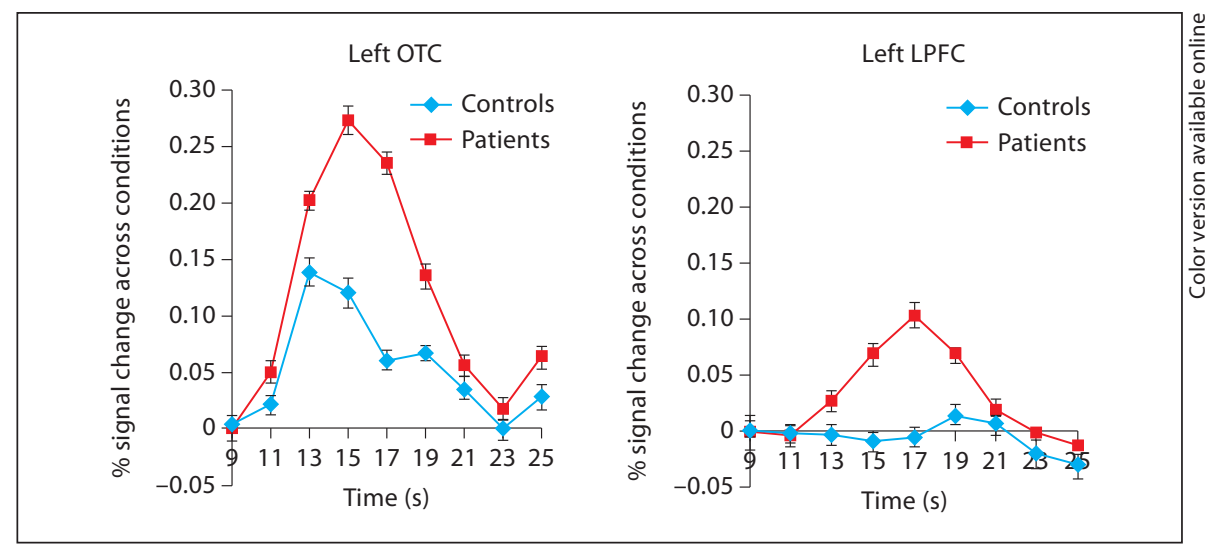

four studies, which could explain why patients did not show the pattern of higher activation observed in the present study. Activation of the left LPFC in patients could also reflect the use of verbal encoding for which left lateralization has been shown [32]. Hyperactivity of the occipital-temporal cortex in patients compared to controls could indicate enhanced encoding and maintenance during WM. Both areas have been shown to be activated for correct versus incorrect responses during encoding and maintenance [33]. Our findings are thus consistent with the evidence that WM needs the interaction between LPFC, temporal and occipital cortex $[34,35]$.

Controls showed a pattern of initial posterior activation in the left OTC, which was followed by right prefrontal activation with a lag of approximately $2 \mathrm{~s}$. This is a common finding of fMRI studies of WM [21] and may correspond to the transfer of information from sensory to prefrontal areas and formation of more stable, abstract representations [8]. Increased activation of the left prefrontal region during the later stages of the task in patients is comparable to the onset of increased activity in the right LPFC in controls. Conversely, patients showed increased and more sustained activity in the OTC compared to controls, starting during the early stages of the task and spanning the maintenance phase. Patients' strategy may thus rely on a more immediate visual representation, conforming to their reports of more vivid mental imagery [36]. Similar supporting mechanisms involving activation of higher visual areas have been reported during a visuospatial task in patients with Alzheimer's disease [37].

A tentative interpretation of the pattern of group differences might thus be that patients achieved similar performance to controls because of increased activity in the left lateral PFC and occipital-temporal region to assist insufficient support by the right lateral and medial prefron-

Working Memory Activity in Schizophrenia tal regions. However, we cannot exclude the possibility that the activation of these areas in patients was unrelated to WM task performance. We observed an emotion-specific decreased WM-related activity for neutral faces in patients compared to controls in the left LFPC, which may indicate that patients need more salient (emotional) stimuli to activate this area to the same degree as controls.

\section{Similar Face WM Performance in Patients and Controls}

WM accuracy decreased significantly with increasing face load in both groups. There were no significant WM performance differences between patients and healthy participants, which are in keeping with the behavioural results of Quintana et al. [23]. However, our results seem to be at odds with the majority of studies with larger sample size, which have reported WM performance deficits for a variety of tasks and stimuli $[1,2,38-40]$. Because of the relatively low power of this study we cannot infer that patients generally do not show a WM deficit (with an effect size for the group difference of $r_{\text {group }}=0.16$, estimated based on the present data, we would have needed 103 subjects for each group to have $80 \%$ power). However, our group of patients only showed a very subtle, if any, performance deficit and is thus interesting for a study of brain mechanisms that potentially support WM processes. Moreover, most of our patients were stable outpatients under treatment at the time of their participation, had a verbal IQ above 100 and a PANSS Cognitive Factor below 8 (online suppl. table 2), indicating low cognitive deficit. They thus represent a relatively homogeneous and highfunctioning subgroup of patients with mainly paranoid schizophrenia.

Several previous studies have attempted to match the performance of patients and controls through compari- 
son of activity at lower WM loads in patients with higher WM loads in controls or by exclusion of incorrect trials from the analysis $[9,10,24,41]$. However, their findings have remained controversial, providing evidence both for and against performance-dependent activity differences between groups in the right PFC.

\section{Can Prefrontal Networks Be 'Trained'?}

Because activity within occipital, temporal, parietal and prefrontal regions has been associated with WM accuracy in healthy controls [42] and patients with schizophrenia $[23,26,41,43]$ interventions that enhance activity in these regions could be particularly effective to improve cognitive functions in patients with schizophrenia. It has been reported repeatedly that patients show an earlier rise of activation in prefrontal regions compared to controls with increasing task difficulty (left shift of the load-response function). Patients' brain activation and performance often break down at lower load levels than in controls $[5,25,44]$. However, the present results show that high-functioning patients may also be able to maintain task-supporting brain activation at high WM loads, although this may be confined to this particular subgroup of mainly paranoid patients without prominent cognitive dysfunction. Wolf et al. [26] have shown that improvement of WM accuracy in patients after multimodal treatment to a level seen in controls correlated with decreased thought disorder and cognitive deficits.

Increased activation of the left LPFC has also been reported during WM in patients treated with an atypical antipsychotic compared to controls, which was also correlated with amelioration of WM performance [45]. Furthermore, improvement of WM performance associated with enhanced frontal activation in patients with schizophrenia has been reported after pharmacological treatment with flumazenil, an inhibitor of GABAergic neurotransmission [46].
However, the beneficial effects of typical and atypical antipsychotics on cognitive functioning appear to be small, vary between cognitive domains, and are influenced by practice effects $[47,48]$. Besides such a neurotransmitter system-based treatment of cognitive deficits the modification of other targets such as neuronal activity-regulated proteins and RNAs involved in neuroplasticity are other areas for potential future development.

The current study was limited by the small sample size. Matching between controls and patients was not perfect although there were no statistically significant differences between both groups. Although the clinical homogeneity of the patient group (paranoid subtype with prominent positive symptoms) may have helped us attain significant group differences even with this relatively small sample, it also constitutes a limitation because findings may not transfer to other types of schizophrenia.

\section{Conclusion}

The results of our study combined with previous findings support a model where hypofrontality in high-functioning patients with paranoid schizophrenia is contrasted with hyperactivity in contralateral homologue areas and sensory areas. Our study also suggests the enhancement of WM-related brain activity as a new target for clinical interventions [49].

\section{Acknowledgements}

We would like to thank all our patients and control participants for their participation, Tony Bedson and his colleagues for their excellent assistance in acquiring the fMRI data. C.W. was supported by the North West Wales NHS Trust and the School of Psychology, Bangor University. D.E.J.L. and M.C.J. are supported by the Biotechnology and Biological Sciences Research Council UK (grant number BB/G021538/1), S.L. was supported by the Wales Institute of Cognitive Neuroscience, and D.E.J.L. and D.H. were supported by the Stanley Medical Research Institute.

\section{References}

1 Lee J, Park S: Working memory impairments in schizophrenia: a meta-analysis. J Abnorm Psychol 2005;114:599-611.

-2 Forbes NF, Carrick LA, McIntosh AM, Lawrie SM: Working memory in schizophrenia: a meta-analysis. Psychol Med 2009;29:889-905.

3 Chen Y, Norton D, McBain R, Ongur D, Heckers S: Visual and cognitive processing of face information in schizophrenia: detection, discrimination and working memory. Schizophr Res 2009;107:92-98.

4 Gooding DC, Tallent KA: Nonverbal working memory deficits in schizophrenia patients: evidence of a supramodal executive processing deficit. Schizophr Res 2004;68:189-201.

5 Manoach DS: Prefrontal cortex dysfunction during working memory performance in schizophrenia: reconciling discrepant findings. Schizophr Res 2003;60:285-298.

6 Honey GD, Fletcher PC: Investigating principles of human brain function underlying working memory: what insights from schizophrenia? Neuroscience 2006;139:59-71.

7 Barch DM: The cognitive neuroscience of schizophrenia. Annu Rev Clin Psychol 2005; 1:321-353.
8 Linden D: The working memory networks of the human brain. Neuroscientist 2007;13: 257-267.

-9 Perlstein W, Carter C, Noll D, Cohen J: Relation of prefrontal cortex dysfunction to working memory and symptoms in schizophrenia. Am J Psychiatry 2001;158:11051113.

10 Thermenos HW, Goldstein JM, Buka SL, Poldrack RA, Koch JK, Tsuang MT, Seidman LJ: The effect of working memory performance on functional MRI in schizophrenia. Schizophr Res 2005;74:179-194. 
$\checkmark 11$ Kay SR, Fiszbein A, Opler LA: The positive and negative syndrome scale (PANSS) for schizophrenia. Schizophr Bull 1987;13:261276.

$\checkmark 12$ Wolf C, Jackson MC, Kissling C, Thome J, Linden DE: Dysbindin-1 genotype effects on emotional working memory. Mol Psychiatry 2011;16:145-155

13 Ekman P, Friesen WV: Pictures of Facial Affect. Palo Alto, Consulting Psychologists Press, 1976

14 Jackson M, Wolf C, Johnston S, Raymond J, Linden D: Neural correlates of enhanced visual short-term memory for angry faces: an FMRI study. PLoS One 2008;3:e3536.

15 Schneider W, Eschman A, Zuccolotto A: EPrime User's Guide. Pittsburgh: Psychology Software Tools Inc., 2002

16 Cowan N: The magical number 4 in shortterm memory: a reconsideration of mental storage capacity. Behav Brain Sci 2001;24: 87-114; discussion 114-185.

17 Talairach J, Tournoux P: Co-Planar Stereotaxic Atlas of the Human Brain. New York, Thieme, 1988.

>18 Gray JR, Braver TS, Raichle ME: Integration of emotion and cognition in the lateral prefrontal cortex. Proc Natl Acad Sci USA 2002; 99:4115-4120.

-19 Rama P, Sala JB, Gillen JS, Pekar JJ, Courtney SM: Dissociation of the neural systems for working memory maintenance of verbal and nonspatial visual information. Cogn Affect Behav Neurosci 2001;1:161-171.

-20 Rama P, Poremba A, Sala JB, Yee L, Malloy M, Mishkin M, Courtney SM: Dissociable functional cortical topographies for working memory maintenance of voice identity and location. Cereb Cortex 2004;14:768-780.

-21 Mayer J, Bittner R, Nikolić D, Bledowski C, Goebel R, Linden D: Common neural substrates for visual working memory and attention. Neuroimage 2007;36:441-453.

-22 Pomarol-Clotet E, Salvador R, Sarro S, Gomar J, Vila F, Martinez A, Guerrero A, OrtizGil J, Sans-Sansa B, Capdevila A, Cebamanos JM, McKenna PJ: Failure to deactivate in the prefrontal cortex in schizophrenia: dysfunction of the default mode network? Psychol Med 2008;38:1185-1193.

23 Quintana J, Wong T, Ortiz-Portillo E, Kovalik E, Davidson T, Marder SR, Mazziotta JC: Prefrontal-posterior parietal networks in schizophrenia: primary dysfunctions and secondary compensations. Biol Psychiatry 2003;53:12-24.

24 Manoach DS, Gollub RL, Benson ES, Searl MM, Goff DC, Halpern E, Saper CB, Rauch SL: Schizophrenic subjects show aberrant fMRI activation of dorsolateral prefrontal cortex and basal ganglia during working memory performance. Biol Psychiatry 2000; 48:99-109.

-25 Karlsgodt KH, Sanz J, van Erp TG, Bearden CE, Nuechterlein KH, Cannon TD: Re-evaluating dorsolateral prefrontal cortex activation during working memory in schizophrenia. Schizophr Res 2009;108:143-150.
26 Wolf RC, Vasic N, Hose A, Spitzer M, Walter $\mathrm{H}$ : Changes over time in frontotemporal activation during a working memory task in patients with schizophrenia. Schizophr Res 2007;91:141-150.

27 Walter H, Wunderlich AP, Blankenhorn M, Schafer S, Tomczak R, Spitzer M, Gron G No hypofrontality, but absence of prefrontal lateralization comparing verbal and spatial working memory in schizophrenia. Schizophr Res 2003;61:175-184.

28 Johnson MR, Morris NA, Astur RS, Calhoun VD, Mathalon DH, Kiehl KA, Pearlson GD A functional magnetic resonance imaging study of working memory abnormalities in schizophrenia. Biol Psychiatry 2006;60:1121

29 Honey GD, Bullmore ET, Sharma T: De-coupling of cognitive performance and cerebral functional response during working memory in schizophrenia. Schizophr Res 2002;53. 45-56.

30 Perlstein WM, Dixit NK, Carter CS, Noll DC, Cohen JD: Prefrontal cortex dysfunction mediates deficits in working memory and prepotent responding in schizophrenia. Biol Psychiatry 2003;53:25-38.

-31 Brahmbhatt SB, Haut K, Csernansky JG, Barch DM: Neural correlates of verbal and nonverbal working memory deficits in individuals with schizophrenia and their highrisk siblings. Schizophr Res 2006;87:191204.

32 Gabrieli JD, Poldrack RA, Desmond JE: The role of left prefrontal cortex in language and memory. Proc Natl Acad Sci USA 1998;95: 906-913.

33 Pessoa L, Gutierrez E, Bandettini P, Ungerleider L: Neural correlates of visual working memory: fMRI amplitude predicts task performance. Neuron 2002;35:975-987.

34 Fuster JM: The prefrontal cortex - an update: time is of the essence. Neuron 2001;30:319333.

35 Curtis CE, D’Esposito M: Persistent activity in the prefrontal cortex during working memory. Trends Cogn Sci 2003;7:415-423.

-36 Sack A, van de Ven V, Etschenberg S, Schatz D, Linden D: Enhanced vividness of mental imagery as a trait marker of schizophrenia? Schizophr Bull 2005;31:97-104.

-37 Prvulovic D, Hubl D, Sack AT, Melillo L, Maurer K, Frölich L, Lanfermann H, Zanella FE, Goebel R, Linden DE, Dierks T: Functional imaging of visuospatial processing in Alzheimer's disease. Neuroimage 2002; 17: 1403-1414.

38 Weinberger E, Cermak L: Short-term retention in acute and chronic paranoid schizophrenics. J Abnorm Psychol 1973;82:220 225.

39 Fleming K, Goldberg TE, Gold JM, Weinberger DR: Verbal working memory dysfunction in schizophrenia: use of a BrownPeterson paradigm. Psychiatry Res 1995;56 155-161.
40 Spindler KA, Sullivan EV, Menon V, Lim KO, Pfefferbaum A: Deficits in multiple systems of working memory in schizophrenia. Schizophr Res 1997;27:1-10.

$\checkmark 41$ Walter H, Vasic N, Hose A, Spitzer M, Wolf RC: Working memory dysfunction in schizophrenia compared to healthy controls and patients with depression: evidence from event-related fMRI. Neuroimage 2007;35: 1551-1561.

42 Haenschel C, Bittner R, Haertling F, Rotarska-Jagiela A, Maurer K, Singer W, Linden D: Contribution of impaired early-stage visual processing to working memory dysfunction in adolescents with schizophrenia: a study with event-related potentials and functional magnetic resonance imaging. Arch Gen Psychiatry 2007;64:1229-1240.

43 Schlosser RG, Koch K, Wagner G, Nenadic I, Roebel M, Schachtzabel C, Axer M, Schultz C, Reichenbach JR, Sauer H: Inefficient executive cognitive control in schizophrenia is preceded by altered functional activation during information encoding: an fMRI study. Neuropsychologia 2008;46:336-347.

44 Callicott JH, Mattay VS, Verchinski BA, Marenco S, Egan MF, Weinberger DR: Complexity of prefrontal cortical dysfunction in schizophrenia: more than up or down. Am J Psychiatry 2003;160:2209-2215.

45 Meisenzahl EM, Scheuerecker J, Zipse M, Ufer S, Wiesmann M, Frodl T, Koutsouleris N, Zetzsche T, Schmitt G, Riedel M, Spellmann I, Dehning S, Linn J, Bruckmann H, Moller HJ: Effects of treatment with the atypical neuroleptic quetiapine on working memory function: a functional MRI followup investigation. Eur Arch Psychiatry Clin Neurosci 2006;256:522-531.

46 Menzies L, Ooi C, Kamath S, Suckling J, McKenna P, Fletcher P, Bullmore E, Stephenson C: Effects of gamma-aminobutyric acidmodulating drugs on working memory and brain function in patients with schizophrenia. Arch Gen Psychiatry 2007;64:156-167.

47 Goldberg TE, Goldman RS, Burdick KE, Malhotra AK, Lencz T, Patel RC, Woerner MG, Schooler NR, Kane JM, Robinson DG: Cognitive improvement after treatment with second-generation antipsychotic medications in first-episode schizophrenia: is it a practice effect? Arch Gen Psychiatry 2007; 64:1115-1122.

48 Keefe RS, Bilder RM, Davis SM, Harvey PD, Palmer BW, Gold JM, Meltzer HY, Green MF, Capuano G, Stroup TS, McEvoy JP, Swartz MS, Rosenheck RA, Perkins DO, Davis CE, Hsiao JK, Lieberman JA: Neurocognitive effects of antipsychotic medications in patients with chronic schizophrenia in the CATIE Trial. Arch Gen Psychiatry 2007;64: 633-647.

49 McGurk SR, Twamley EW, Sitzer DI, McHugo GJ, Mueser KT: A meta-analysis of cognitive remediation in schizophrenia. Am J Psychiatry 2007;164:1791-1802. 